\title{
Al margen del antisemitismo: Respuestas del semanario Mundo Judío al Movimiento Nacional Socialista de Chile (1935-1938) ${ }^{1}$
}

On the margins of Antisemitism: Responses of the weekly paper Mundo Judio to the Chilean Movimiento Nacional Socialista (1935-1938)

\author{
Gustavo Guzmán \\ gu.guzman@gmail.com \\ Universidad de Chile \\ Chile
}

\section{RESUMEN}

Durante los años treinta del siglo pasado, se produjo en Chile una proliferación de agrupaciones nacionalistas de derecha contrarias al liberalismo, la democracia y el comunismo. En algunos casos, tales agrupaciones adoptaron también el fascismo y el antisemitismo provenientes de Europa, desarrollando campañas antijudías inéditas en la Historia de Chile. El presente artículo indaga en las respuestas del semanario Mundo Judío, principal órgano de prensa de la colectividad judía chilena de los años treinta, frente al antisemitismo del Movimiento Nacional Socialista (MNS) de Jorge González von Marées, el grupo fascista chileno más importante de los años treinta, distinguiendo dos principales formas de reacción.

PALABRAS ClAVE: Antisemitismo en Chile - Mundo Judío - Movimiento Nacional Socialista de Chile - nacismo chileno - prensa sionista de Chile

\begin{abstract}
During the thirties many right-wing nationalist groups appeared in Chile. Most of them declared to be against Liberalism, Democracy and Communism. In some cases these groups also embraced the Fascism and the Antisemitism coming from Europe, developing anti-Semitic campaigns, unprecedented in the Chilean History. This article enquires in the responses of the weekly paper Mundo Judio, main press publication of the Chilean Jewish community during the
\end{abstract}

\footnotetext{
${ }^{1}$ El presente artículo sintetiza parte de los capítulos 4 y 5 de "La patria sin judíos: Antisemitismo nacionalista en Chile, 1932-1940. Los casos del Movimiento Nacional Socialista y del Partido Nacional Fascista", tesis para acceder al grado de Magíster en Historia, mención Historia de Europa, de la Universidad de Chile.
} 
thirties, to the Antisemitism of the Jorge González von Marées' Movimiento Nacional Socialista, the most important Chilean fascist group of the period, distinguishing two main reactions.

KEYWORDS: Antisemitism in Chile - Mundo Judio - Chilean Movimiento Nacional Socialista - Chilean nacismo - Chilean Zionist press

Pocos meses después de la "República Socialista" de Marmaduque Grove -cuando un grupo de oficiales de izquierda de la Fuerza Aérea ejecutó un Golpe de Estado que los puso en el poder por doce días-y de su propio nacimiento como partido, el joven Movimiento Nacional Socialista de Chile (MNS) definió el materialismo que inspiraba a la izquierda como "fruto de las doctrinas del judío Karl Marx” y reveló a sus seguidores que “el comunismo no es, como generalmente se cree, un simple movimiento ideológico" sino "la lucha de una raza por el dominio mundial". Para los nacistas $^{2}$, liderados por el carismático Jorge González von Marées, el comunismo era "un movimiento genuinamente judío" cuya finalidad no consistía en "destruir el régimen capitalista" sino en "conquistar el dominio político del mundo para la raza judía". Estas sensacionales revelaciones continuaron días después, cuando "La Página Nacional Socialista" del diario El Imparcial denunció que los judíos no sólo controlaban el comunismo sino también el capitalismo, "el cinematógrafo, la gran prensa, la banca y la política" (El Imparcial, 18 noviembre 1932; 25 noviembre 1932; 2 diciembre 1932).

Expresiones antisemitas como estas, inéditas en la Historia de Chile, se repitieron una y otra vez durante los años treinta, dotando al MNS de un perfil ideológico similar al de sus pares fascistas europeos e instalando en el país un novedoso antisemitismo secular. El presente artículo indaga en las reacciones que tales expresiones suscitaron en el semanario Mundo Judio, publicación oficial de la Federación Sionista de Chile y principal órgano de prensa judío de la época, para lo cual ha sido dividido en tres partes. La primera da cuenta del antisemitismo del MNS,

\footnotetext{
${ }^{2}$ Como una forma de diferenciarse del nazismo alemán, el MNS se hizo llamar 'nacismo' y a sus miembros, 'nacistas'. 
deteniéndose en sus características más significativas y en el estado de la cuestión respecto de su estudio; la segunda se refiere a la situación del judaísmo chileno de comienzos de los años treinta, particularmente de la prensa comunitaria; finalmente, la tercera parte expone las principales reacciones de Mundo Judio frente al antisemitismo nacista, distinguiendo dos principales formas de respuesta.

\section{El antisemitismo del Movimiento Nacional Socialista de Chile (1932-1938)}

Durante las últimas décadas se ha producido un creciente interés académico por el nacionalismo chileno de los años treinta, particularmente por el Movimiento Nacional Socialista, publicándose una cantidad significativa de estudios sobre el nacismo. Resumidamente, podemos decir que la bibliografía en torno al MNS ha centrado su atención preferentemente en cuestiones como su similitud con los modelos fascistas europeos (Edwards Vives y Frei Montalva, 1949; Donoso, 1954; Pike, 1963; Fontaine, 1974; Bicheno, 1972; Ramírez Necochea, 1978; Etchepare y Stewart, 1995); en su carácter de movimiento propiamente fascista (Sznajder, 1990; Deutsch, 1996; Klein, 2000); en su ambigüedad ideológica (Deutsch, 2005); en la composición social de sus militantes (Etchepare y Stewart, 1995) y en las causas de su fracaso (Sznajder, 1990; Deutsch, 1996; Klein, 2000). Sus manifestaciones antisemitas, en cambio, se mantuvieron en un lugar secundario hasta comienzos de los años noventa, según veremos.

El consenso académico actual, al cual adscribimos, es que el MNS fue un movimiento propiamente fascista que, inspirado por sus símiles europeos, intentó construir un proyecto político de tercera vía y un espacio propio en el abigarrado sistema de partidos chileno de los años treinta, para lo cual dispuso de giros y variaciones ideológicas que por momentos lo acercaron a la derecha conservadora y por momentos a una izquierda populista, sin comprometer significativamente su carácter de movimiento fascista. Del mismo modo, tales variaciones afectaron sus expresiones antisemitas.

Como decíamos, el antisemitismo nacista se mantuvo como un tema de escaso interés en la bibliografía especializada hasta comienzos de los años noventa. Hasta ese momento, los estudios 
historiográficos sobre el MNS se limitaron a describir algunas de las expresiones antijudías aparecidas en Trabajo, dedicándoles en el mejor de los casos algunas líneas y comentarios, aunque sin someterlas a un análisis exhaustivo ni establecer su lugar preciso dentro de la ideología nacista (Potashnik, 1974). En algunos casos, el tema fue derechamente soslayado (Grugel, 1985; Etchepare y Stewart, 1995). Esta situación cambió con los trabajos del profesor chileno-israelí Mario Sznajder (1990, 1992, 1994), primer autor en poner el antisemitismo nacista en el centro de su análisis historiográfico. En dichos trabajos, Sznajder establece, en primer lugar, que el antisemitismo del MNS "no era de carácter racial biológico como el del nazismo alemán", sino que recurría a otros elementos más bien ligados a la visión spengleriana de la historia, que atribuía a los judíos una esencia materialista verificable tanto en el liberalismo como en el comunismo. En segundo lugar, que dicha visión abría la puerta al uso de imágenes conspirativas sobre el judaísmo, opción que el nacismo no tardó en tomar: la integridad chilena era atacada simultáneamente por judíos comunistas, desde la izquierda, y por judíos capitalistas, desde la derecha. Y, en tercer lugar, que esta imagen conspirativa permitía entrelazar antisemitismo y antiimperialismo por medio del uso de un "mito movilizador" - en términos sorelianos ${ }^{3}$ - que tendía un puente entre realidad cotidiana y el etéreo imperio internacional encabezado por el judío. Este mito movilizador antisemita permitía superar las diferencias políticas y de clases encerrando "en un solo actor social todo lo negativo, tanto del capitalismo cuanto del comunismo" (Sznajder, 1990, 50-1; 1994, 101-2).

Años después, Sznajder añadió a estas conclusiones que el auge del fascismo en Europa, en particular del nazismo alemán, habría sido más determinante en el desarrollo del antisemitismo nacista que el antijudaísmo tradicional católico y que el eco que encontraron estas representaciones antisemitas se debió, en gran medida, a la grave crisis económica y política que Chile había experimentado desde 1929. Es en este marco que el autor analiza el "fuerte incremento del antisemitismo" en Chile durante los años treinta. Las diatribas que el nacismo lanzó desde una publicación de cierto alcance popular como Trabajo estuvieron influenciadas por la propaganda nazi alemana y por las teorías conspirativas antisemitas de los Protocolos de los Sabios de Sión, que acusaban a los judíos de querer dominar el mundo por medio de una

\footnotetext{
${ }^{3}$ Para Sorel el mito social era un recurso de movilización de masas que en vez de apelar al intelecto, como la conciencia de clase marxista, apela a los sentimientos de las masas. Carece de una lógica interna coherente, priorizando su valor estético y emocional (Sznajder, 1994, 96-7).
} 
conspiración cuyos tentáculos alcanzaban y dominaban el comunismo y el capitalismo internacionales. Estas representaciones, de tono antimaterialista y anticosmopolita, constituían argumentaciones de carácter socio-económico que pretendían explicar los males de Chile en base a una nefasta influencia judía (Sznajder, 2007, 82-5).

El segundo gran aporte al estudio del antisemitismo nacista fue hecho por la historiadora estadounidense Sandra McGee Deutsch, durante la segunda mitad de los años noventa (1996). Como destaca la autora, entre 1932 y 1937 el nacismo recurrió con regularidad a representaciones antisemitas con el objetivo de capitalizarlas políticamente, siendo el período entre 1935 y 1937 el de mayor intensidad. Sin embargo, desde mediados de 1937 éstas desaparecieron casi por completo. Incluso en el período de mayor actividad antisemita, ésta se redujo al periódico Trabajo y, en mucho menor medida, a la revista Acción Chilena, sin alcanzar los documentos oficiales y programáticos del partido ${ }^{4}$. Deutsch ve estas variaciones en el antisemitismo nacista como respuestas a los cambios en el escenario político chileno de los años treinta y a los intentos del partido de adaptarse a ellos. Según la autora, uno de los elementos distintivos de la alternativa a la izquierda que el MNS pretendió levantar a mediados de los años treinta fue precisamente este discurso antisemita, el cual supo encajar con elementos antiimperialistas y anticapitalistas de modo de mostrarse radical en su lucha con la izquierda. El hecho de que estuviera en boga entre los fascistas europeos, la necesidad de encontrar chivos expiatorios para los efectos de la Gran Depresión en Chile y el aumento demográfico de la población judía en Chile durante los años treinta explicarían, según Deutsch, la decisión del MNS de adoptar esta campaña antisemita (Deutsch, 1996, 161-8).

La importancia de esta campaña radica, según Deutsch, en que habría intentado sustituir la lucha de clases por la denuncia del "capitalismo judaico", y de este modo arrebatar a la izquierda parte de su electorado. Asimismo, la teoría de la conspiración judía mundial, elemento fundamental de la campaña antisemita nacista, asomaba como un medio promisorio de atraer seguidores al partido, pues la identificación del judaísmo con fuerzas que amenazaban Chile podría estimular sentimientos nacionalistas y antiimperialistas. Por ello, Deutsch cree que el antisemitismo se

\footnotetext{
${ }^{4}$ Esta es la razón por la que nuestro trabajo se centra exclusivamente en el periódico Trabajo, soslayando otras fuentes primarias nacistas.
} 
convirtió efectivamente en "una parte integral de la alternativa nacista a la izquierda" y en una estrategia de movilización popular, al menos durante el período 1935-1937. La situación cambió a partir de 1937, cuando los diputados nacistas se ubicaron junto a la izquierda en contra de Alessandri. Desde entonces, el MNS se mostró menos ansioso que antes por distinguirse de la izquierda y sustituirla. Por lo demás, era evidente que los resultados de la campaña antijudía del MNS distaban de ser positivos. Por último, pone de relieve que a pesar de su agresividad, el antisemitismo nacista nunca traspasó el límite de lo representativo para llegar a la violencia física (Deutsch, 1996, 171-2).

Por último, vale la pena destacar el aporte de Marcus Klein (2000) al estudio del antisemitismo nacista. Klein establece una relación directa entre los giros ideológicos efectuados por el movimiento a lo largo de los años treinta y el espacio que el antisemitismo tuvo en cada una de estas etapas. Durante la etapa inicial del partido (1932-1933), la campaña antisemita nacista habría tenido como objetivo principal ampliar la base de seguidores del movimiento, considerando el éxito que el nazismo alemán conseguía en esos días sobre la base de la utilización política del antisemitismo. Para ello, el MNS apeló a estereotipos tradicionales que relacionaban a los judíos con el dinero, además de la asociación judaísmo/comunismo señalada al comienzo de este artículo. Respecto de la segunda etapa del partido (1933-1935), Klein considera que el antisemitismo "no llegó a convertirse en un tema dominante del nacismo, posiblemente porque la propaganda no encontró la respuesta esperada en un país con una colectividad judía particularmente pequeña". Aunque ataques antisemitas aparecieron una y otra vez en las páginas de Trabajo, esta campaña careció de consistencia, por lo que prefiere considerarlos como "arranques ocasionales" más que como una actitud consistente basada en los Protocolos de los Sabios de Sión. Y respecto de la tercera etapa del nacismo (1935-1937), concluye que si bien recurrió al antisemitismo como una forma de atacar al Frente Popular y a la izquierda, a costa de quienes pretendió hacerse de un espacio político propio, tales ataques aparecían de manera lo suficientemente irregular e inconsistente como para describirlos como "una parte integral de la alternativa nacista a la izquierda y una estrategia de movilización popular", como pretende Deutsch. Por último, en la cuarta etapa del partido (1937-1938), el antisemitismo fue abandonado casi por completo (Klein, 2000, 123-35). 
Ahora bien, ¿de qué manera representaban los nacistas chilenos a los judíos? La representación más utilizada por Trabajo durante los años treinta mostraba a los judíos como creadores y controladores del comunismo, el que utilizaban para dominar a los gentiles, en general, y a los chilenos, en particular. Así, junto con expresiones como las reproducidas al comienzo de este escrito, podemos encontrar numerosas manifestaciones en el sentido de que "el comunismo es, lisa y llanamente, obra del malévolo y corruptor judaísmo internacional" (Trabajo, 20 abril 1933). Entre 1935 y 1937, esta asociación judaísmo/comunismo se utilizó sobre todo para atacar al Frente Popular -conglomerado político de centro izquierda compuesto por radicales, socialistas y comunistas, principalmente- e intentar así arrebatarles votos. De este modo, no sorprende que a mediados de 1936 el periódico nacista denunciara que el Frente Popular perseguía la instauración de una dictadura del proletariado, "vale decir, la tiranía de una pequeña minoría judaicomasónica" (Trabajo, 19 mayo de 1936).

Del mismo modo, el periódico nacista acusaba a los judíos de crear y dominar el capitalismo en beneficio propio. En este sentido, resultan paradigmáticos el artículo "Nuestra industrial salitrera agoniza bajo las garras del judaísmo internacional", que denunciaba oscuros manejos por parte de la familia judía Guggenheim, y la editorial "El gran culpable", que advertía que si bien el “imperialismo judeo-comunista" representaba un peligro enorme para Chile, el "imperio judeocapitalista" ya era una realidad, pues había convertido la economía chilena en un apéndice del sistema económico norteamericano (Trabajo 27 julio 1933; 10 mayo 1934). Nuevamente, el período en que la asociación judaísmo/capitalismo se dio con mayor fuerza fue entre $1935 \mathrm{y}$ 1937. Esto quedó de manifiesto sobre todo a mediados de 1936, cuando el Ministro de Hacienda de Alessandri y futuro candidato presidencial de la derecha, Gustavo Ross Santa María, visitó Londres para negociar la venta de la Compañía Chilena de Tabacos. Entonces, el semanario nacista denunció que "no sólo el salitre y el cobre han pasado a manos del imperialismo judaico, sino que todas nuestras industrias". Paralelamente, dado que durante su estadía en Londres Ross Santa María visitó a miembros de la famosa familia judía de banqueros Rothschild, Trabajo aprovechó de titular su número del 22 de mayo de 1936 “El Sanhedrín Internacional, inspirador de la política de Ross". En dicho número se acusaba al Ministro de Hacienda de haber viajado a 
la capital inglesa con el único objeto de "implorar la bendición papal del pontífice del judaísmo internacional" y entregar las riquezas nacionales "a ese sanhedrín de judíos internacionales". Durante este período incluso González von Marées, que por lo general se abstenía de emitir comentarios personales en contra de los judíos, se refirió a la visita de Ross Santa María a los Rothschild en la editorial "A merced del imperialismo judaico", calificándola como "verdadera traición a los intereses nacionales" (Trabajo, 9 mayo 1936; 22 mayo 1936; 23 mayo 1936).

Pero la conspiración judía mundial denunciada por el periódico nacista, bajo la evidente inspiración de los Protocolos de los Sabios de Sión, no se limitaba al dominio del capitalismo y el comunismo. Según Trabajo, ella incluía también otros elementos propios de la modernidad, como la "cinematografía yanqui-judía", que permitía difundir antivalores que destruían los conceptos básicos de moral y decencia. En otra clara muestra de la influencia de los Protocolos, los nacistas creían que los judíos controlaban en su beneficio a la masonería (Trabajo, 15 junio 1933; 24 mayo 1934; 20 marzo 1937).

Pese a las similitudes entre el antisemitismo nacista y el nazi alemán, Trabajo se defendió en más de una ocasión diciendo que su antisemitismo no era racista, a diferencia de aquél. Esta idea incluso es avalada por Mario Sznajder, para quien "el antisemitismo del MNS no era de carácter racial biológico como del nazismo alemán" (Sznajder, 1990, 50-1). En la editorial "El Movimiento Nacional Socialista y los judíos”, el periódico nacista explicaba su aversión por el judaísmo en que éste dedicaba "su habilidad a explotar todas las fallas de nuestros sistemas económicos" más que por cuestiones raciales. Era por ello que prefería una inmigración como la alemana, cuyos miembros "honrados y laboriosos" producían "inmensos beneficios a la colectividad nacional" (Trabajo, 1 junio 1933).

Otro de los tópicos antisemitas desarrollado con fuerza por Trabajo durante los años treinta fue la denuncia de una "invasión judía" que amenazaba a Chile. Tal como las representaciones antes señaladas, ésta comenzó en 1933 - primer año de vida del periódico nacista Trabajo- pero alcanzó su mayor regularidad y agresividad entre 1935 y 1937. Entonces, abundaron textos donde se denunciaba que los judíos estaban ingresando en cantidades desproporcionadas al país, haciéndose de un lugar preponderante en el comercio, las profesiones liberales y la 
administración pública. Según Trabajo, los judíos "sólo se dedican al comercio, siendo que en Chile sobran los intermediarios y faltan brazos para la agricultura y la minería" y "se organizan para encarecer los artículos de consumo, desplazando así al elemento nacional". La invasión judía a Chile llegaba a tal punto, afirmaba el periódico nacista, que incluso un nieto del Presidente Alessandri era hijo de padre judío y sería circunciso en los días venideros (Trabajo 18 abril 1935; 17 junio 1936; 18 junio 1936).

\section{Sobre la colectividad judía de Chile de los años treinta y el semanario Mundo Judío}

A comienzos de los años treinta, Chile tenía una población aproximada de 4,2 millones de habitantes, de los cuales no más de 4.000 eran judíos. En su mayoría, eran askenazíes provenientes de Rusia, Polonia y Rumania, así como sefaradíes de Macedonia, Salónica y Turquía. Con la llegada de Hitler al poder, en 1933, la composición de esta pequeña colectividad comenzó a cambiar, dada la creciente llegada de askenazíes desde Europa central. Desde el punto de vista organizativo, no existía mayor unidad entre los grupos que componían la colectividad. Con excepción de la Federación Sionista -fundada en 1919, con el impulso de la Declaración Balfour- no existía una institución central o unitaria que aglutinase al judaísmo chileno. Esta federación estaba compuesta por una generación de judíos jóvenes con estudios universitarios, en su mayoría nacidos en Chile o llegados en su infancia, que a diferencia de sus padres se mostró muy activa desde el punto de vista político. Fueron precisamente estos jóvenes quienes encabezaron la defensa del judaísmo de los ataques antisemitas provenientes del fascismo chileno y lo hicieron desde la tribuna ofrecida por la incipiente prensa comunitaria (Nes-El, 2009, 51-5).

En general, la generación de judíos mayores se mostró reacia a participar activamente en política. Como suele ocurrir con los inmigrantes de primera generación, este grupo poco asimilado prefirió abocarse a sus labores privadas y reunirse con sus pares en actividades sociales y religiosas. En el caso puntual de los askenazíes, éstos publicaron dos semanarios en yiddish, $E l$ Semanario Israelita de Chile y La Prensa Israelita, publicaciones que informaban preferentemente sobre las actividades sociales y religiosas del grupo de habla yiddish pero que no intentaron influir en la opinión pública chilena, dada la opción lingüística recién señalada. En tal 
sentido, desconocemos cuál fue su reacción frente al nacimiento de un Movimiento Nacional Socialista en Chile, en abril de 1932, pues de haberlo mencionado en sus páginas lo hicieron en yiddish, renunciando a la posibilidad de llamar la atención de la opinión pública chilena sobre el particular y de movilizarla en su contra, a diferencia de lo obrado por la generación de judíos jóvenes desde las páginas de Mundo Judio. En tal sentido, cabe destacar que las únicas oportunidades en las que El Semanario Israelita de Chile y La Prensa Israelita incluyeron secciones en español -sin considerar los avisos comerciales, por cierto- se produjeron en abril y mayo de 1933, cuando el primero de ellos publicó sendos números especiales dedicados a la situación de sus correligionarios a manos del nazismo alemán (El Semanario Israelita de Chile, 15 abril 1933; 23 mayo 1933).

Desde el punto de vista de la generación de judíos jóvenes, la publicación de prensa más importante de comienzos de los años treinta fue la revista Nosotros, dirigida por el futuro diputado socialista Natalio Berman ${ }^{5}$. Tras su desaparición, en 1934, dicho lugar fue ocupado por el semanario Mundo Judio, publicación oficial de la Federación Sionista de Chile y uno de los órganos de prensa más importantes de la historia del judaísmo chileno. A diferencia de $E l$ Semanario Israelita de Chile y La Prensa Israelita, Mundo Judio se publicaba en español, era vendido en quioscos y tenía un tiraje relativamente alto, lo que le permitió ser leído también por un público no judío y alcanzar una considerable influencia en la opinión pública de la época (Nes-El, 2009, 55).

En su primer número, aparecido los primeros días de 1935, Mundo Judío declaró tener una doble finalidad. La primera, "informar sobre todo lo que ocurre en la vida judía a través del mundo", en particular sobre la situación de los judíos en Alemania, donde los nazis "han convertido el antisemitismo en sistema de gobierno". La segunda, defender a los judíos orientando a "la opinión pública del país frente a los ataques que se hagan del judaísmo". Al respecto, el semanario sionista adelantaba que impediría que se "envenenen las almas jóvenes de América con informaciones malévolas sobre nuestra existencia" (Mundo Judio, 3 enero 1935).

\footnotetext{
${ }^{5}$ Lamentablemente, la Biblioteca Nacional no cuenta con ejemplares de Nosotros correspondientes al período 19321934, por lo que desconocemos su reacción frente al nacimiento de un Movimiento Nacional Socialista en Chile, en abril de 1932.
} 
La importancia de esta segunda finalidad de Mundo Judio quedó de manifiesto a poco andar. Un mes después de su aparición, el semanario sionista se refirió a las acusaciones nacistas en contra del "judaísmo internacional" y sus supuestas maniobras por medio de la familia minera Guggenheim, del empresario Agustín Edwards Mac Clure y del Ministro de Hacienda Gustavo Ross Santa María, a pesar de que estos dos últimos ni siquiera eran judíos. Según Mundo Judío, los nacistas intentaban instalar artificialmente el problema judío en Chile sobre la base de relacionar al "pueblo o nación judía con la interdependencia mundial originada por el actual sistema capitalista", ignorando "los elementos interconfesionales que rigen en los negocios financieros" y sentando "una premisa absolutamente falsa" respecto del judaísmo (Mundo Judío, 21 febrero 1935).

Como veremos en las próximas páginas, la decisión de responder directa y explícitamente a las provocaciones nacistas se mantendría a lo largo de todo el primer año de vida de Mundo Judío 1935-, haciendo que en numerosas ocasiones el semanario sionista denunciara el intento nacista de instalar en Chile el "problema judío". Sin embargo, dicha elección se vería modificada a partir de 1936, año en que la campaña antisemita del MNS alcanzó su punto más álgido, dando lugar a una nueva estrategia.

\section{Respuestas de Mundo Judio al antisemitismo nacista (1935-1938)}

A lo largo de 1935, primer año de vida de Mundo Judío y período en el que el antisemitismo de Trabajo comenzó a hacerse más persistente y agresivo, las respuestas del semanario sionista a las provocaciones nacistas fueron numerosas y persistentes. Así, durante el mes de mayo asistimos a intervenciones como las de "Chanteclair" - columnista que destacaría en más de una ocasión por sus polémicas con los nacistas criollos-, quien denunció la forma maliciosa en que Trabajo presentaba las naturalizaciones de judíos residentes en Chile, mostrándolas como una grave amenaza para el país. Según este columnista, el MNS no sólo buscaba atacar a los judíos sino también al gobierno de Alessandri, por lo que rechazaba de modo tajante "la pretensión de los nacistas que desean mezclarnos en sus asuntos de política local” (Mundo Judio, 2 mayo 1935). 
Dado que los nacistas negaban ser antisemitas, Chanteclair volvió a polemizar con ellos en octubre de 1935, recordando "los gritos contra los judíos que se oyen en sus asambleas y las vociferaciones callejeras de los vendedores de su órgano oficial que insultan dos veces a la semana $^{6}$ al judaísmo que califican de internacional”. En la oportunidad también se refirió a los permanentes intentos del MNS de vincular judaísmo con comunismo y de hacer pagar al judaísmo en su conjunto por la obra de individuos puntuales, estando "siempre dispuestos a calumniar al judaísmo o imponerle normas, como si el judío, al igual que todo hombre civilizado, no tuviese el más perfecto derecho de obrar personalmente conforme a los dictados de su esclarecida conciencia" (Mundo Judio, 31 octubre 1935).

Este rechazo al intento de hacer pagar al judaísmo en su conjunto por la conducta de individuos puntuales se repitió en más de una ocasión durante el primer año de vida de Mundo Judío. Así, frente a la acusación de que "la casi totalidad de los mercaderes de la Bolsa Negra son judíos" esgrimida por los nacistas, el semanario sionista declaró que no aceptaba que las responsabilidades personales fueran achacadas al conjunto de los judíos de Chile ni que se usara la palabra 'judío' con una "acepción denigrante legada por prejuicios" (Mundo Judio, 2 mayo 1935).

El rol de Jorge González von Marées en la campaña antisemita de su partido también fue tratado por Mundo Judio a fines de 1935. En la oportunidad, el periodista Moisés A. Riesemberg acusó al "Jefe" nacista de tener una actitud ambivalente respecto a la "cuestión judía", pues en una entrevista concedida al propio Riesemberg para "The Jewish Telegraphic Agency" de Nueva York el líder nacista se habría mostrado contrario a la persecución antisemita del nazismo alemán, dado que "en sus propias venas corre sangre semita, porque su abuela era judía". Sin embargo, mientras para el extranjero "condena las persecuciones de los judíos", en Chile basaba su programa político en el odio a los mismos (Mundo Judio, 5 diciembre 1935).

\footnotetext{
${ }^{6}$ La primera publicación del MNS fue "La Página Nacional Socialista", publicada por el diario derechista santiaguino El Imparcial entre 1932. En 1933, el partido comenzó a publicar su propio semanario, Trabajo, que en 1935 ya se publicaba dos veces por semana. Finalmente, a partir de 1936 Trabajo se convirtió en diario.
} 
Además de estas polémicas, durante sus primeros trece meses de vida Mundo Judio denunció por lo menos en tres ocasiones haber recibido amenazas directas por parte de miembros del MNS, lo que nos recuerda que la hostilidad de los nacistas chilenos por los judíos iba más allá de lo meramente discursivo (Mundo Judio, 14 febrero 1935, 26 diciembre 1935, 30 enero 1936).

Por todo lo anterior, Mundo Judio recibió con alegría la formación de la Liga para la Defensa de la Cultura por parte de un grupo de destacados intelectuales nacionales -que incluía a Augusto D’Halmar, Mariano Latorre, Marta Brunet y Manuel Rojas- a fines de 1935. La Liga se declaraba contraria a la destrucción de libros y a la persecución antisemita en Alemania, así como a la "anulación de derechos individuales incorporados a la vida universal" (Mundo Judio, 12 diciembre 1935). La cercanía de Mundo Judío con agrupaciones antifascistas como la Alianza de Intelectuales de Chile y el Instituto Antirracista de Chile se acrecentaría a fines de los años treinta, a medida que la situación europea empeoraba y que el antisemitismo del fascismo chileno - encarnado en el Partido Nacional Fascista de Raúl Olivares Maturana, tras el colapso del MNS a fines de 1938- se agudizaba, sin embargo dicho período excede el marco cronológico de este trabajo.

A pesar de las respuestas dadas por el semanario sionista a las provocaciones del MNS, no todos sus colaboradores estaban de acuerdo con la idea de trabar polémica con Trabajo, de contestar directa y explícitamente a sus ataques. En su columna "Al margen del antisemitismo", Marcos Weinstein defendió una postura totalmente contraria e incluso ironizó con que hubiera judíos "entregados a la tarea de combatir el antisemitismo con argumentos y razones". Según Weinstein, resultaba inútil el diálogo con los nacistas porque "el respeto a la verdad no existe para el antisemitismo. Si fuera necesario negar la luz del día, se negaría sin pudor", por eso "ningún judío verdaderamente inteligente lo ha hecho en el pasado próximo o lejano" (Mundo Judío, 2 mayo 1935).

La evidencia recogida de las fuentes primarias utilizadas en este artículo sugiere que la postura planteada por Weinstein habría terminado imponiéndose al interior de la Federación Sionista de Chile y de los encargados de su semanario Mundo Judio, al menos desde comienzos de 1936. A 
pesar de que, como vimos en la primera parte de este artículo, durante 1936 el antisemitismo nacista alcanzó su punto de mayor intensidad y agresividad -baste recordar los ataques contra Ross Santa María por su visita a los Rothschild en Londres-, el semanario sionista no respondió siquiera una vez a sus provocaciones. Evidentemente, este soslayo de las provocaciones nacistas en el momento más álgido de su campaña antisemita no pudo ser casualidad, sino que supuso la implementación de una nueva estrategia de contención, basada en ignorar las expresiones antijudías del MNS y evitar así que éstas encontraran resonancia en la opinión pública gracias a tales polémicas.

Llamativamente, la única ocasión en la que Mundo Judío volvió a referirse al antisemitismo en Chile durante el período cubierto por este artículo se produjo a mediados de 1937, cuando el MNS había abandonado la campaña antisemita de los años previos. Entonces, el semanario sionista publicó una serie de tres artículos titulada "El antisemitismo en Chile", de Yedi Brannover. En la primera parte, el autor vaticinaba escaso éxito a las campañas antijudías desarrolladas en América Latina y en particular en Chile, dada la escasa población judía residente. De todos los grupos nacionalistas existentes en Chile, no obstante, se debía tener particular cuidado con el MNS, pues "ha sido sólo el nacismo el que sostiene una táctica antisemita". En la segunda parte, el autor se refería a los medios propagandísticos que "el antisemitismo chileno emplea para su mejor difusión", entre los cuales se encontraban libros como Los Protocolos de los Sabios de Sión y El Judio Internacional de Henry Ford y periódicos como Trabajo. Por último, en la tercera parte, Brannover enumeraba las acusaciones que esta campaña antisemita hacía a los judíos: en primer lugar, tenían planeado dominar Chile económica y políticamente por medio del capitalismo internacional - encarnado en la familia minera Guggenheim- y de la masonería; en segundo lugar, se apoderaban de actividades fundamentales como la medicina y el comercio; en tercer lugar, eran incapaces de desempeñarse como agricultores; y, en cuarto lugar, eran incapaces de asimilarse a la nación chilena, manteniéndose ajenos a ella. Obviamente, todas estas acusaciones eran refutadas y replicadas con sólidos argumentos por parte del autor (Mundo Judio, 29 julio 1937; 5 agosto 1937; 19 agosto 1937).

Con excepción de esta serie de artículos publicados a mediados de 1937, entre febrero de 1936 y diciembre de 1938 el semanario Mundo Judio no volvió a referirse una sola vez al MNS ni a 
responder a sus provocaciones. Esta nueva estrategia con la que la Federación Sionista enfrentó al nacismo chileno sería puesta a prueba a mediados del 1939, con la aparición del semanario La Patria, publicación oficial del Partido Nacional Fascista de Chile (PNF), el grupo político más radicalmente antisemita de la historia de Chile, pero cuya acción escapa al marco cronológico de este artículo.

\section{Conclusiones}

Como se desprende del material revisado, la campaña antisemita emprendida por el MNS a mediados de los años treinta no pasó inadvertida para la colectividad judía chilena de la época. La generación de judíos jóvenes, con educación universitaria y políticamente activos, encabezó la reacción de los judíos chilenos en contra del antisemitismo nacista y lo hizo principalmente bajo la forma de la denuncia pública, desde las páginas del semanario sionista Mundo Judío, en contra de las maniobras del MNS. La denuncia en contra de la campaña antisemita del MNS ocupó un lugar importante en las páginas de Mundo Judio, donde sólo las informaciones sobre la labor sionista en Palestina y la situación de los judíos alemanes tuvieron mayor cobertura.

Sin embargo, la manera en que enfrentó la campaña antisemita del MNS varió a lo largo del tiempo. Durante su primer año de vida -1935- Mundo Judío se mostró muy activo y respondió explícitamente a los intentos nacistas de instalar en Chile la "cuestión judía". Así, replicó y entró en polémica en varias ocasiones con el periódico Trabajo para desacreditar sus alegatos antisemitas. Sin embargo, a partir de 1936 la estrategia para enfrentar las injurias del MNS cambió notablemente: a pesar de que durante ese período -al menos hasta el primer tercio de 1937- la campaña antisemita del partido de González von Marées alcanzó su punto más crítico, Mundo Judio hizo como si éste no existiera, dejando de mencionarlo por completo. Vale decir, se impuso la idea de Marcos Weinstein de no estimular la persistencia de tal campaña sobre la base de polémicas, la idea de mantenerse "al margen del antisemitismo". 
En todo caso, tanto la denuncia y la polémica con los editores de Trabajo como la nueva estrategia consistente en ignorar las provocaciones nacistas, incluso en su período de mayor regularidad y persistencia, ponen de manifiesto el impacto de la campaña antisemita del MNS: el tema fue lo suficientemente importante para que Mundo Judio lo recogiera como un tema de importancia durante su primer año de vida y para que, dada su relevancia y la posibilidad de que se viera fortalecido por las eventuales polémicas, se decidiera implementar la nueva estrategia basada en el silencio. En cualquiera de ambos casos, el antisemitismo del que hizo gala el MNS entre su nacimiento y su giro a la izquierda, a mediados de 1937, no pasó desapercibido ni desapareció tras el giro izquierdista. Sería recogido, desarrollado y profundizado por uno de los principales sucesores del MNS, el Partido Nacional Fascista de Chile. Aunque, ciertamente, ese ya es otro tema. 


\section{Bibliografía}

Fuentes primarias

"La Página Nacional Socialista" de El Imparcial, julio 1932 - marzo 1933

Trabajo, abril 1933 - septiembre 1938

Acción Chilena, enero 1934 - febrero 1938

Semanario Israelita de Chile, enero 1930 - julio 1933

La Prensa Israelita, agosto 1933 - mayo 1936

Mundo Judio, enero 1935 - diciembre 1938

Fuentes secundarias

Bicheno, H. (1972). Anti-parliamentary themes in Chilean History, 1920-1970. Government and Opposition, 7 (3), 351-388.

Cohn, N. (1983). El mito de la conspiración judía mundial. Los Protocolos de los Sabios de Sión. Madrid: Alianza.

Deutsch, S. M. (1996). Anti-Semitism and the Chilean Movimiento Nacional Socialista. En Sheinin, D. y Barr, L. B. (Eds.). The Jewish Diaspora in Latin America: New Studies on History and Literature (pp. 161-181). Nueva York: Garland.

Deutsch, S. M. (2005). Las derechas. La extrema derecha en la Argentina, el Brasil y Chile. 1890-1939. Buenos Aires: Universidad Nacional de Quilmes Editorial.

Donoso, R. (1954). Alessandri: agitador y demoledor. México: Fondo de Cultura Económica.

Edwards Vives, A. y Frei Montalva, E. (1949). Historia de los partidos políticos chilenos. Santiago: Pacífico. 
Etchepare, J. y Stewart, H. (1995). Nazism in Chile: A Particular Type of Fascism in South America. Journal of Contemporary History, 30 (4), 577-605.

Fontaine, A. (1974). Ideas nacionalistas chilenas. En Arce Eberhard, A. y Campos Menéndez, E. (Eds.). Pensamiento nacionalista (pp. 233-247). Santiago: Gabriela Mistral.

Grugel, J. (1985). Nationalists Movements and Fascist Ideology in Chile. Bulletin of Latin American Research, 4 (2), 109-122.

Klein, M. (2000). A Comparative Analysis of Fascist Movements in Argentina, Brazil and Chile between the Great Depression and the Second World War. Tesis doctoral, Universidad de Londres, Londres, Gran Bretaña.

Nes-El, M. (2009). La actitud de Chile frente a la inmigración judía durante la Segunda Guerra Mundial (1933-1943). En Nes-El, M. Estudios sobre el judaísmo chileno (pp. 51-66). Jerusalén: Revista de Oriente y Occidente.

Nes-El, M. (2009). Los judíos y su actuación en la política chilena, 1920-1952. En Nes-El, M. Estudios sobre el judaísmo chileno (pp. 67-89). Jerusalén: Revista de Oriente y Occidente.

Pike, F. (1963). Chile and the United States, 1880-1962: The Emergence of Chile's Social Crisis and the Challenge to the United Stated Diplomacy. Notre Dame: University of Notre Dame Press.

Potashnik, M. (1974). Nacismo: National Socialism in Chile, 1932-1938. Tesis doctoral, Universidad de California, Los Ángeles, Estados Unidos.

Ramírez Necochea, H. (1978). El fascismo en la evolución política de Chile hasta 1970. Araucaria de Chile, 1, 9-33. 
Sznajder, M. (1990). El Movimiento Nacional Socialista: Nacismo a la chilena. Estudios Interdisciplinarios de América Latina y el Caribe, 1 (1), 41-57.

Sznajder, M. (1992). El nacionalsocialismo chileno de los años treinta. Mapocho, 32, 169-193.

Sznajder, M. (1994). Impacto del mito movilizador: de racismo verbal a genocidio. En Gurevich, B. y Escudé, C. (Eds.) El genocidio ante la historia y la naturaleza humana. (pp. 91-117). Buenos Aires: Universidad Torcuato Di Tella.

Sznajder, M. (2007). Diferentes trayectorias del antisemitismo en Chile. Índice 37 (24), 77-99. 